NOTA CIENTÍFICA

\title{
Extensión de la distribución del rango migratorio de la "Dormilona de Frente Negra", Muscisaxicola frontalis (Aves: Tyrannidae) en el Perú
}

\section{Extension of the distribution of the migratory range of the "Black-fronted Ground Tyrant" Muscisaxicola frontalis (Aves: Tyrannidae) in Peru}

\author{
Renzo Alcocer ${ }^{1}$, Grace P. Servat ${ }^{2}$ y Wilfredo Mendoza $^{3}$
}

1 Área de Ornitología, Colección Científica, Museo de Historia Natural, Universidad Nacionat San Agustin de Arequipa. Arequipa, Peri. Email: renzopaulehotmail. Perú. Email: renzopaul@hotmail. com

2 Smithsonian Conservation Biology Institute, CCES-NZP; 1100 Jefferon Drive, Suite 3139, hington, DC 20013-7012. Email: grace.servat@gmail.com

3 Laboratorio de Florística, Museo de Historia Natural-UNMSM. Av. Arenales 1256, Jesús María ima Perú gmail.com

Presentado: $\quad 27 / 07 / 2011$ Aceptado: $\quad$ 03/11/2011 Publicado online: 08/02/2012

\begin{abstract}
Resumen
Extendemos el rango migratorio, altitudinal y de distribución norte de Muscisaxicola frontalis ("Dormilona de Frente Negra"), migrante Austral previamente reportado para el sur del país. La especie fue observada en laderas pedregosas cerca a remanentes de bosque de Queñoa (Polylepis flavipilla, Rosaceae) a $4450 \mathrm{~m}$ de
\end{abstract} altitud, en el Departamento de Huancavelica.

Palabras clave: Muscisaxicola frontalis, distribución, Huancavelica, ladera rocosa, bosque de Queñoa, migratoria Austral.

\section{Abstract}

We extent the migratory range of distribution and elevation of Muscisaxicola frontalis (Black-fronted Groundtyrant), Austral migrant reported previously only in the Southern region of the country. The species was observed in rocky slopes near Polylepis flavipilla (Rosaceae) woodlands at $4450 \mathrm{~m}$ of altitude, in the Huancavelica Department.

Keywords: Muscisaxicola frontalis, distribution, Huancavelica, rocky slopes, Polylepis woodlands, Austral migrant.
La Dormilona de Frente Negra (Muscisaxicola frontalis (Burmeister, 1860), Tyrannidae), esta distribuida en regiones alto andinas (> $2900 \mathrm{~m}$ de altitud) desde Santiago de Chile hasta Antofagasta (Chile) y de Mendoza hasta Río Negro (oeste de Argentina). En invierno migra hasta Jujuy (noroeste de Argentina) llegando hasta el Altiplano de Bolivia (> 3600 m) (Fjeldså et al. 1990) (Fig. 1a). En el Perú, M. frontalis ha sido registrada en periodo no reproductivo (Plenge 2010), desde abril a septiembre, en los Departamentos de Arequipa, Moquegua y Puno (Fig. 1b, 1c), de 3750 a $4300 \mathrm{~m}$ de altitud, donde se le encuentra asociada con pastizales, humedales, laderas pedregosas y rocosas (Fjeldså y Krabbe 1990, Jaramillo et al. 2003, Schulenberg et al. 2007).

Un individuo de $M$. frontalis fue observado el 17 de mayo del 2010, durante una visita al remanente de bosque de Queñoa (Polylepis flavipila (Bitter) M. Kessler \& Schmidt-Leb., Rosaceae), en el área cercana a la localidad de Huaytará, en el kilómetro 153 de la carretera Los Libertadores-Wari (Departamento de Huancavelica, Provincia de Huaytará, 13³0'08.01"S; 7509'22.60"W, $4450 \mathrm{~m}$ ) (Fig. 1c). El individuo fue observado posado sobre el suelo de una ladera pedregosa, con vegetación dispersa constituida principalmente por Jarava ichu (Poaceae), arbustos de Senecio spp., Chuquiraga spinosa (Asteraceae) y pequeños arbolillos de $P$. flavipila. El individuo presentaba coloración negra en la región frontal que se prolongaba hasta la corona o región pileal central que contrastaba con la coloración gris pálida de la región pileal lateral y la región loral de color blanco (Fig. 2).

El 18 de mayo, capturamos un individuo mediante el uso de redes de neblina, el cual fue preparado como ejemplar de estudio para su posterior identificación. El estado del plumaje, la coloración definida del pileo y lorum; Además, de la ausencia de color ante (canela claro) en los bordes de las plumas cobertoras alares (Jaramillo et al. 2003), revelan de que se trata de un individuo macho adulto (testes $1 \times 1 \mathrm{~mm}$ ), aunque presenta
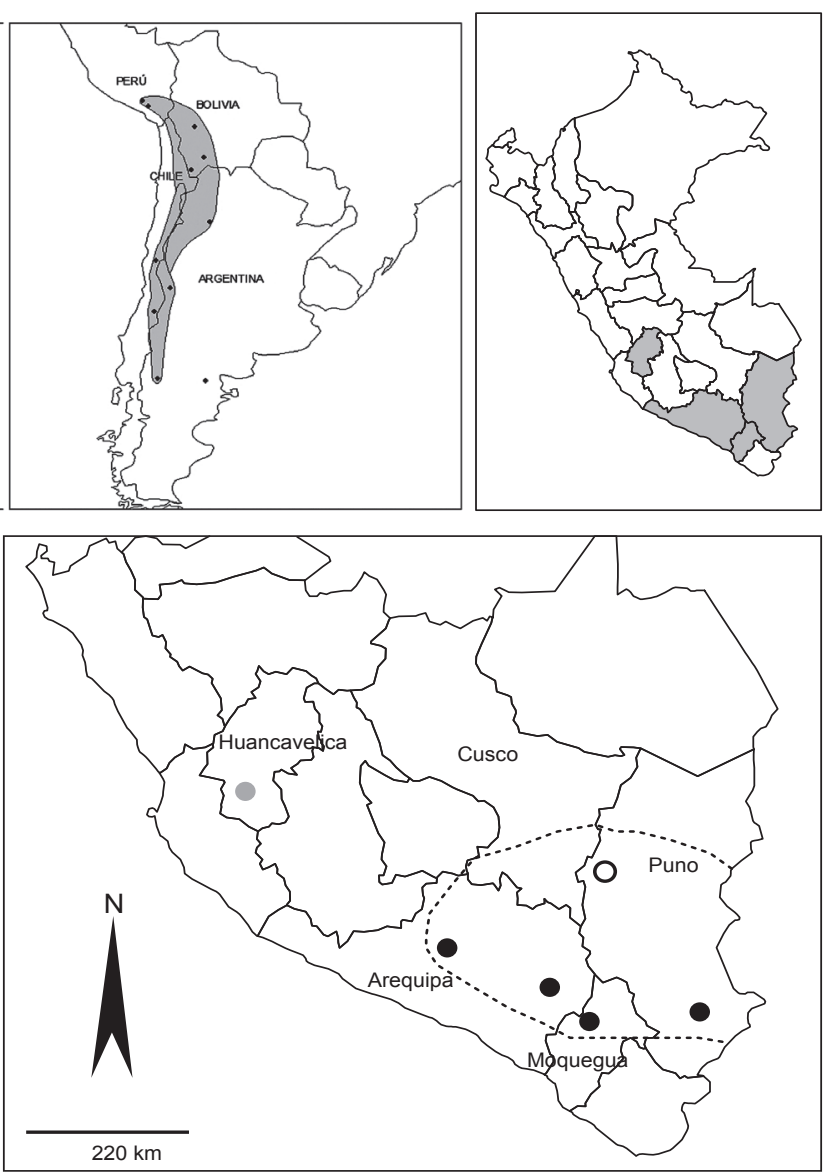

Figura 1. Distribución de Muscisaxicola frontalis en Sudamérica (a) (Ridgely, et al. 2007) y en el Perú (b y c). En c) Los círculos negros corresponden a registros confirmados, los círculos blancos son registros por confirmar (Schulenberg et al. 2006). El círculo gris representa el nuevo reporte para la localidad de Huaytará (Huancavelica). 


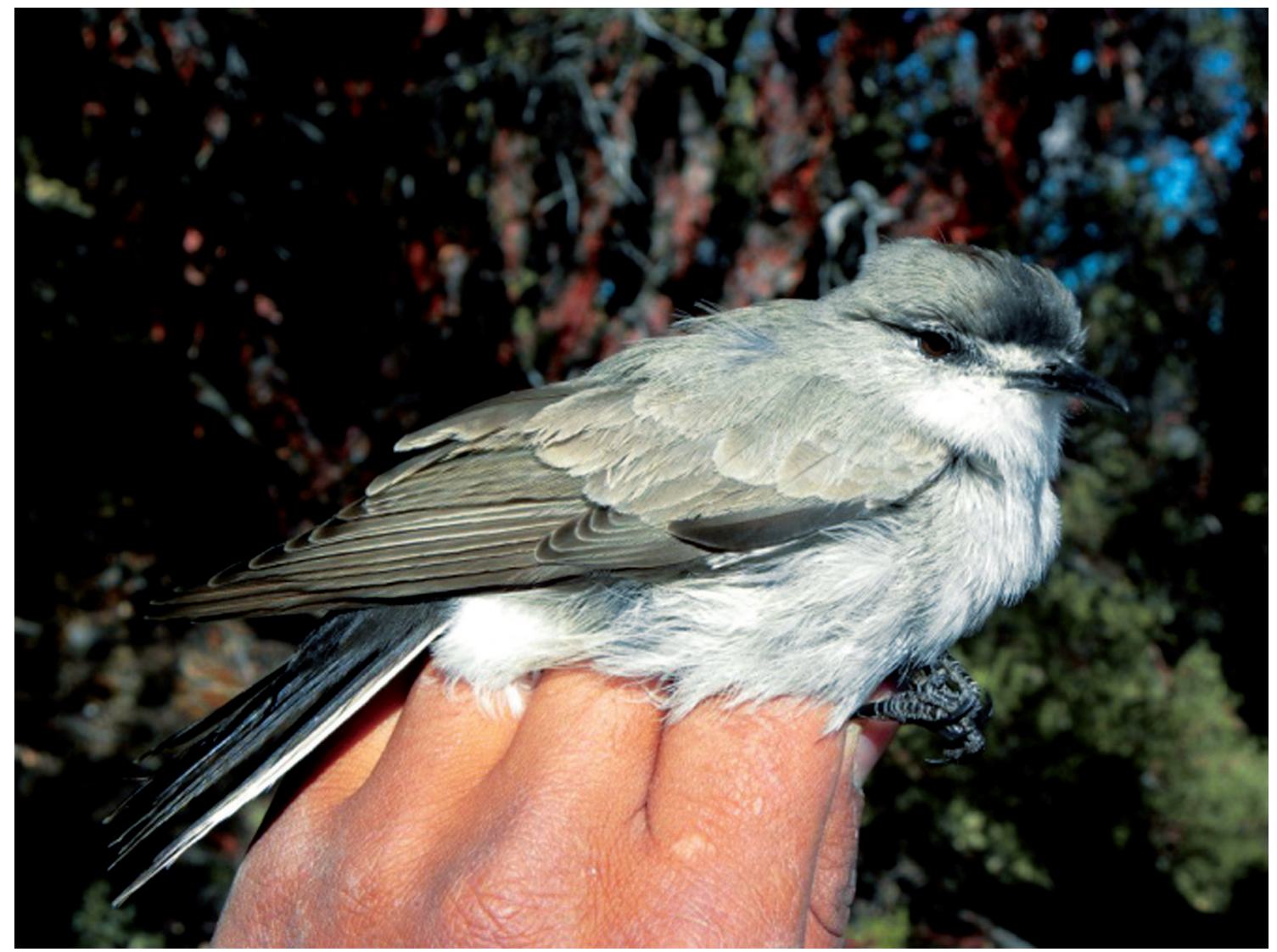

Figura 2. Muscisaxicola frontalis, nótese la coloración negra en la región frontal que se prolonga hasta la región pileal central (corona) y la región loral de color blanco (Foto: R. Alcocer).

un porcentaje de osificación del cráneo del $25 \%$. Una posterior revisión de la literatura y comparación con especímenes de las colecciones ornitológicas de los Museos de Historia Natural de la Universidad Nacional de San Agustín de Arequipa (MUSA) y de la Universidad Nacional Mayor de San Marcos (MUSM) nos permitieron confirmar que se trata de la especie $M$. frontalis. El espécimen (junto con el contenido estomacal y tejidos preservados) se encuentra depositado en la colección del MUSM (GSV 2010-85). Con este reporte extendemos el rango migratorio de distribución norte de la especie hasta Huancavelica y su rango altitudinal hasta los $4450 \mathrm{~m}$ para el Perú.

\section{Agradecimientos}

Agradecemos a E. López Tejada, Director del Museo de Historia Natural de la Universidad de San Agustín de Arequipa (MUSA) y a L. Salinas, Curadora de la Colección Ornitológica del Museo de la Universidad Nacional Mayor de San Marcos (MUSM) por facilitarnos el acceso a las colecciones a su cargo. Así mismo agradecemos a las autoridades respectivas de la Dirección General de Gestión Forestal y de Fauna Silvestre por el permiso otorgado para el presente estudio (RD No. 0130-2010-AG-DGFFS-DGEFFS del 26/3/2010).

\section{Literatura citada}

Fjeldså J. \& N. Krabbe. 1990. Birds of the High Andes. Copenhagen. Zoology Museum, University of Copenhagen and Svendborg, Apollo Books.
Gonzales N., H. Zeballos P. \& E. López T. 2001. Aves del Valle del Colca y de la Reserva de Salinas y Aguada Blanca. Proyecto Araucaria Valle del Colca. AECI. Arequipa.

INRENA. 2001. Plan Maestro de la Reserva de Salinas y Aguada Blanca, Instituto Nacional de Recursos Naturales, Ministerio de Agricultura. Arequipa

Jaramillo, A., P. Burke \& D. Beadle. 2003. Field Guide to the Birds of Chile, Christopher Helm, London.

Plenge, M. A. 2010. Lista de las Aves de Perú. Lima, Perú. $<$ https:// sites.google.com/site/boletinunop/checklist $>$ (acceso 27/07/2010)

Remsen, J. V., Jr., C. D. Cadena, A. Jaramillo, M. Nores, J. F. Pacheco, J. Pérez-Emán, M. B. Robbins, F. G. Stiles, D. F. Stotz \& K. J. Zimmer. 2010. A classification of the bird species of South America. American Ornithologists' Union. $<$ http://www.museum.lsu.edu/ Remsen/SACCBaseline. html $>$ (acceso 27/07/2010)

Ridgely, R. S., T. F. Allnutt, T. Brooks, D. K. McNicol, D. W. Mehlman, B. E. Young, and J. R. Zook. 2007. Digital Distribution Maps of the Birds of the Western Hemisphere, version 3.0. NatureServe, Arlington, Virginia, USA.

Schulenberg, T. S., D. F. Stotz \& L. Rico. 2006. Distribution maps of the birds of Peru, version 1.0. Environment, Culture \& Conservation (ECCo). The Field Museum of Natural History. $<$ http://fm2.fieldmuseum.org/uw_test/birdsofperu $>$ (acceso 27/07/2010)

Schulenberg T., D. F. Stotz, D. F. Lane, J. P. O’Neill \& T. A. Parker III. 2007. Birds of Peru. Princeton Field Guides. 